SUPPORTING INFORMATION

Regulating Gene Expression in Zebrafish Embryos Using LightActivated, Negatively Charged Peptide Nucleic Acids

XinJing Tang, ${ }^{\dagger}$ Shingo Maegawa, ${ }^{\ddagger}$ Eric S. Weinberg, ${ }^{\ddagger}$ and Ivan J. Dmochowski*, ${ }^{\dagger}$

${ }^{\dagger}$ Department of Chemistry, University of Pennsylvania, 231 South $34^{\text {th }}$ Street, Philadelphia, PA 19104-6323;

${ }^{\ddagger}$ Department of Biology, University of Pennsylvania, 204E Carolyn Lynch Laboratory, Philadelphia, PA 19104

Telephone: (215) 898-6459, Fax: (215) 898-2037, Email: ivandmo@sas.upenn.edu 


\section{In situ hybridization}

In situ hybridization procedures were performed as previously described, with BM purple alkaline phosphatase substrate (Roche) used as the chromogen in these experiments. ${ }^{1}$ For hybridization, embryos were incubated at $65{ }^{\circ} \mathrm{C}$ overnight with the RNA probe. Probes were used to detect gsc and otx2 genes (mRNA) for bozozok and chordin experiments, respectively. Figure S1 shows the in situ hybridization results for otx2 involving PNA-chd experiments under different injection conditions.

\section{Synthesis of light-activated, negatively charged PNAs}

Synthesis of the light-activated, negatively charged PNAs was performed according to a previous report, with minor modifications. ${ }^{2}$ Sense 2'-OMe RNA was dissolved in $0.1 \mathrm{mM}$ DTT, 0.05 M TEAA buffer overnight, then passed through a NAP-10 column, and purified by RPHPLC (0-30\% acetonitrile in $0.05 \mathrm{M}$ TEAA buffer in $30 \mathrm{~min}$ at $\left.25^{\circ} \mathrm{C}\right)$.

Negatively charged peptide nucleic acid (ncPNA, used without purification as received from Active Motif, Carlsbad, CA) was first dissolved in $200 \mu \mathrm{L}$ of $0.1 \mathrm{M}$ phosphate buffer (pH 7.2) and warmed to $60^{\circ} \mathrm{C}$. The photocleavable linker, PL, in DMF was added. The reaction mixture was incubated at $35^{\circ} \mathrm{C}$ for $40 \mathrm{~min}$, the oligonucleotide (ncPNA-PL conjugate) was prepurified with a NAP-10 column and purified by RP-HPLC (5-30\% acetonitrile in 0.05 M TEAA buffer at $\left.40{ }^{\circ} \mathrm{C}\right)$.

After mixing the sense 2'-OMe RNA and antisense (ncPNA-PL) strands and concentrating, the solution was passed through a NAP-10 column and left to stand at room temperature for 30 min before addition of phosphate buffer $(0.1 \mathrm{M}, \mathrm{pH} 7.2)$ and sodium chloride solution $(0.3 \mathrm{M})$ to reduce the possibility of $G$ quadruplex formation of the G-rich 2'-OMe-RNA sense strand. After 
standing at room temperature overnight, the mixture was passed through a NAP-10 column to remove the salts, and the final ncPNA-PL-sRNA conjugate (caged ncPNA) was purified by HPLC using a Resource Q anion exchange column (buffer A, 0.02 M phosphate buffer, 5 M urea, $15 \%$ acetonitrile, $\mathrm{pH}$ 7; buffer $\mathbf{B}, \mathbf{A}+2 \mathrm{M} \mathrm{NaCl} ; \mathbf{B}, 5-45 \%$ in $40 \mathrm{~min}$ at $40{ }^{\circ} \mathrm{C}, 2 \mathrm{~mL} / \mathrm{min}$, Figure S2). Mass, caged PNA-chd: calculated (M/Z) 9921.4, MALDI-TOF 9927.9; photolysis product 1, calculated 5325.2, MALDI-TOF 5307.8; photolysis product 2, calculated 4554.2 (-44 for $\mathrm{CO}_{2}$ ), MALDI-TOF 4547.4; mass, caged PNA-boz: calculated (M/Z) 8575.9, MALDI-TOF 8559.0.

Melting temperatures of ncPNA/sRNA duplex and ncPNA-PL-sRNA conjugate (caged ncPNA)

The ncPNA/sRNA duplex and ncPNA-PL-sRNA conjugate (caged ncPNA) were dissolved in $10 \mathrm{mM}$ phosphate buffer ( $\mathrm{pH}$ 7.0) at $1 \mu \mathrm{M}$ concentration and melting studies were carried out with a DU 800 UV-Vis spectrophotometer (Beckman DU 800). Samples were monitored at 260 $\mathrm{nm}$ while heating or cooling at a rate of $1{ }^{\circ} \mathrm{C} / \mathrm{min}$. Melting temperatures were determined from the peak of the first derivative plot of $\mathrm{Abs}_{260}$ vs. temperature. The melting temperatures $\left(T_{\mathrm{m}}\right)$ of the PNA-chd/sRNA and PNA-boz/sRNA duplexes were $70{ }^{\circ} \mathrm{C}$ and $39{ }^{\circ} \mathrm{C}$, respectively (Figure S3). However, the melting temperature $\left(T_{\mathrm{m}}\right)$ of caged PNA-chd was at least $90{ }^{\circ} \mathrm{C}$, for caged PNA-boz, the $T_{\mathrm{m}}$ was $80{ }^{\circ} \mathrm{C}$.

\section{Measurement of molecular beacon fluorescence}

A molecular beacon (MB) with black hole quencher (BHQ1) and fluorescein (FAM) was designed to be complementary to the PNA-chd antisense strand. Experiments with $40 \mathrm{nM} \mathrm{MB}$ solution were performed in $10 \mathrm{mM}$ phosphate buffer (pH 7.0) and the same solution containing 2 
eq. native PNA-chd, caged PNA-chd, or caged PNA-chd that had been UV-irradiated for 8 min (UV transilluminator, $9 \mathrm{~mW} / \mathrm{cm}^{2}$ at $365 \mathrm{~nm}$ ). Fluorescence spectra were collected for $\mathrm{MB}, \mathrm{MB}+$ native PNA-chd, $\mathrm{MB}+$ caged PNA-chd, and $\mathrm{MB}+$ caged PNA-chd +8 min photolysis at $30{ }^{\circ} \mathrm{C}$, respectively (Figure S4). This competition between PNA-chd/sRNA (inactive) and PNAchd/mRNA (active) duplex forms was also assayed by comparing the fluorescence intensity of a molecular beacon ${ }^{3}$ when native PNA-chd, caged PNA-chd, or uncaged PNA-chd was present in solution. Fluorescence from the beacon in the presence of caged PNA-chd increased roughly 2fold upon UV irradiation. When the beacon was mixed with native PNA-chd, the fluorescence intensity was yet 4 -fold higher. This suggested that only $\sim 25 \%$ of the uncaged PNA-chd hybridized to the beacon.

\section{Zebrafish breeding and embryo collection}

Two male and one female adult zebrafish were separated by a divider in a special breeding tank the night before fertilization and subsequent collection of embryos from the tank. At 9:30 am the following morning, the divider was removed, which allowed the male and female zebrafish to mate. After 15-20 min, the fertilized embryos were collected, washed, and transferred to a Petri dish filled with 10\% Hank’s saline solution.

\section{Microinjection of ncPNA and caged ncPNA and UV irradiation of embryos}

In order to determine whether low-level UV light had adverse effects on early development, we irradiated 50 zebrafish (Danio rerio) embryos at 3 hours post fertilization (3 hpf) for 0, 5, 10, and 20 min with a UV transilluminator (Spectroline Ultraviolet transilluminator, TL-365R, 9 $\mathrm{mW} / \mathrm{cm}^{2}$ at peak intensity, $365 \mathrm{~nm}$ ), and compared the phenotypic results at 24 hpf. No differences between zebrafish were observed, even after 20 min UV irradiation. The duration of 
UV illumination required for complete photocleavage was determined through separate experiments involving Cy3-labeled ncPNA-PL-sDNA constructs in buffer and in zebrafish embryos (Figure S5). We injected some embryos with ncPNA-PL-sDNA-Cy3, followed by UV photolysis for $8 \mathrm{~min}$. Then the supernatant of the embryo suspension was subject to denaturing polyacrylamide gel with in vitro control. The native ncPNA or caged ncPNA was microinjected (using a Harvard Apparatus microinjector, Zeiss Stemi SV11 microscope, Narashige micromanipulator) into the cytoplasm of 1- or 2-cell zebrafish embryos (typically $0.5 \mathrm{mM}$ stock solution, $10 \mathrm{~nL}$ injected per embryo). The injected embryos, as well as uninjected (control) embryos, were incubated at $28^{\circ} \mathrm{C}$. At 2 hpf (for bozozok) and 3 hpf (for chordin), half of the embryos injected with caged PNA were irradiated for 8 min with a UV transilluminator (Spectroline Ultraviolet transilluminator, TL-365R, $9 \mathrm{~mW} / \mathrm{cm}^{2}$ at peak intensity, $365 \mathrm{~nm}$ ). The remaining embryos were cultured without exposure to any light.

\section{Confocal images of zebrafish embryos at $24 \mathrm{hpf}$}

After microinjection and photolysis, the embryos were incubated in the dark at $28{ }^{\circ} \mathrm{C}$. At 24 hpf, the chorion of each zebrafish was removed with forceps by gently making a tear in the chorion and releasing the embryo. The zebrafish embryos were then transferred to another culture dish with glass coverslip bottom for imaging by inverted confocal laser scanning microscope (Olympus FV1000) at room temperature. A UPlan S-Apo 4×, 0.16 NA objective lens (Olympus 1-U2B822) was used to image the entire embryo. The images were collected in transmitted light mode.

Native PNA-chd solutions over the concentration range 0-0.5 $\mathrm{mM}$ were injected into zebrafish embryos at the 1- or 2-cell stage. At $24 \mathrm{hpf}$, the embryos showed a corresponding range 
of phenotypes: the phenotype was concentration-dependent as shown with representative embryos in Figure S6.

Caged PNA-chd and caged PNA-boz were also injected into the zebrafish embryos at the 1or 2-cell stage. After 2-3 hpf, UV light was used to irradiate the embryos, and phenotype was observed at 24 hpf for activated caged PNA-chd and caged PNA-boz as shown in Figure 2 of the text and Figure S7.

Figure S1. Effect of PNA-chd on otx2 expression at mid-gastrula stage (8 hpf). Embryos are aligned such that the vegetal pole points up and the animal pole points down. A) Uninjected wild-type embryos showed strong otx2 expression at arrow. B) Injection of PNA-chd (0.1 mM) resulted in reduction of ot 2 mRNA. C) Injection of caged PNA-chd $(0.5 \mathrm{mM})$ did not affect otx2 expression. D) UV uncaging of PNA-chd $(0.5 \mathrm{mM})$ at $3 \mathrm{hpf}$ caused reduction of otx2 expression.

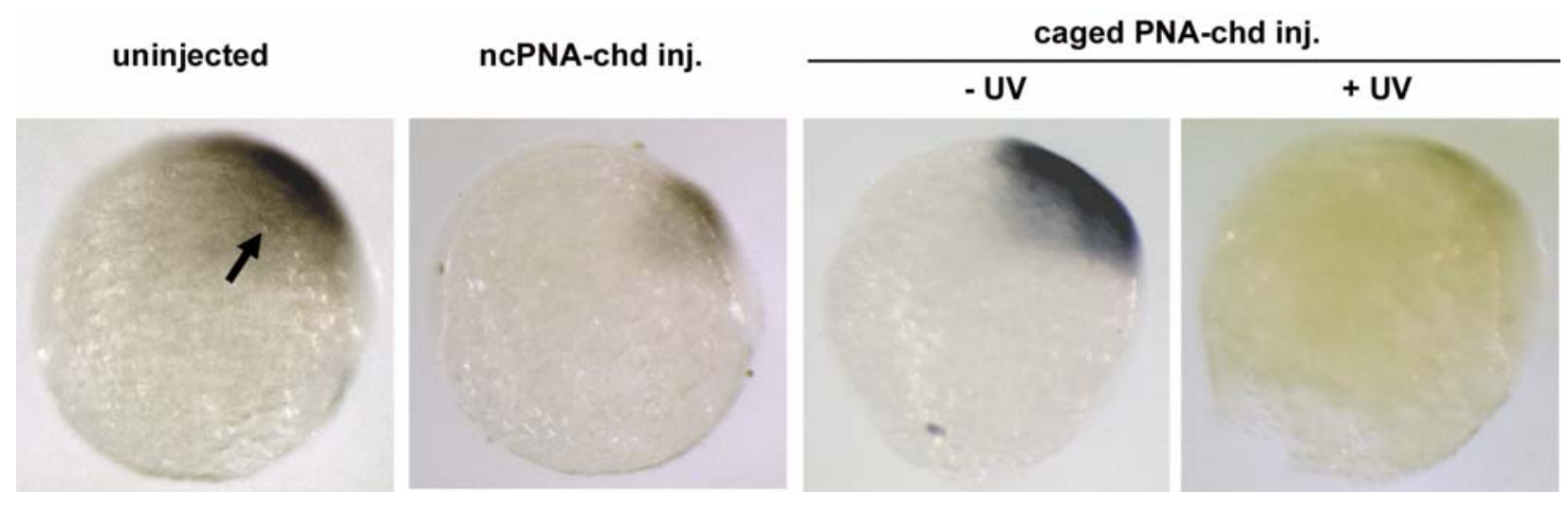


Figure S2. HPLC traces of duplex of PNA-chd/sRNA, caged PNA-chd, caged PNA-boz and ncPNA-PL-sDNA-Cy3. Gradient of $\mathbf{B}, 5 \rightarrow 45 \%$ in $40 \mathrm{~min}$ at $40{ }^{\circ} \mathrm{C}, 2 \mathrm{~mL} / \mathrm{min}$ flow rate. Major fraction containing the desired product was collected, as indicated with vertical lines.
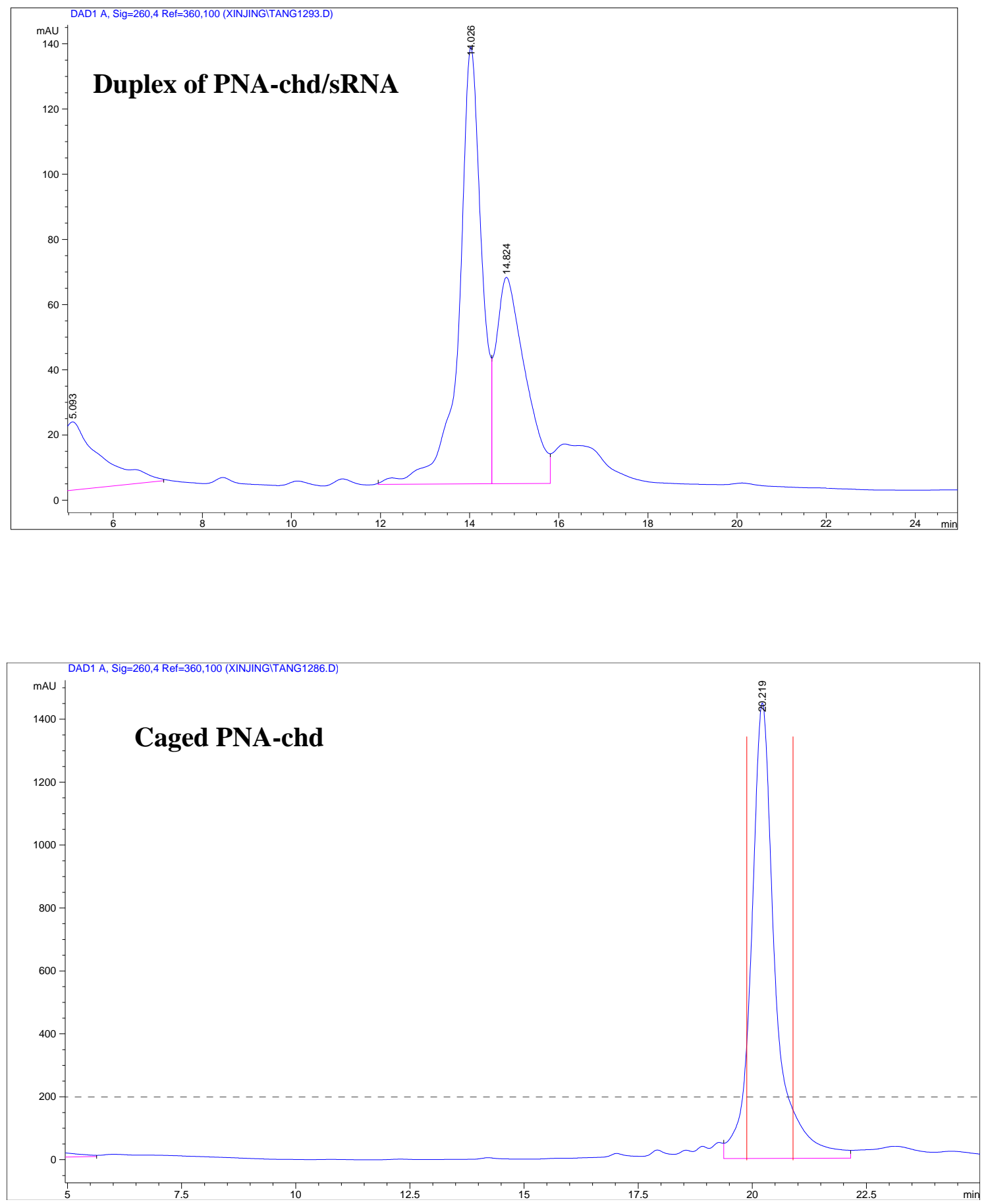

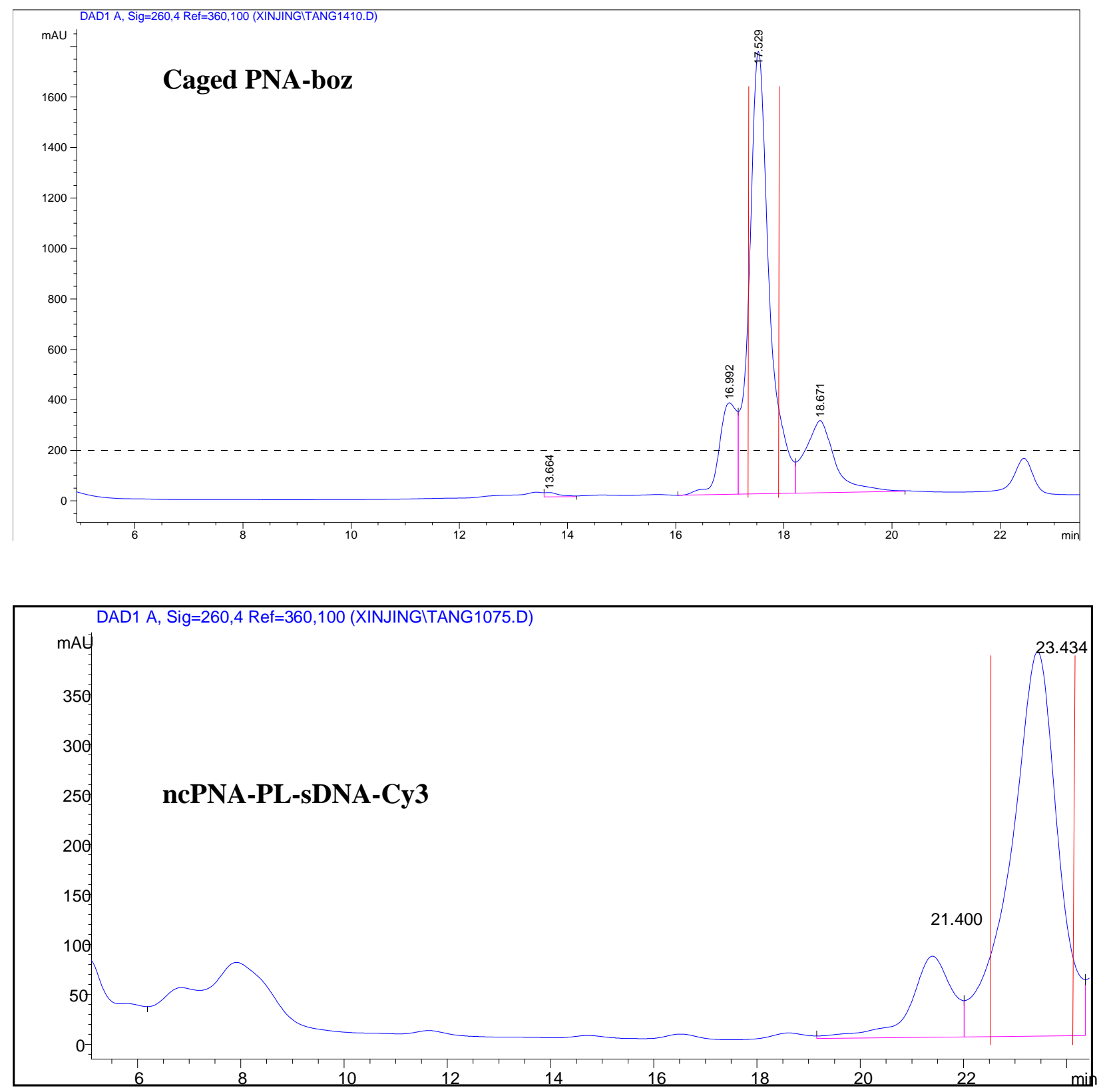
Figure S3. Melting curve for ncPNA/sRNA duplexes and caged PNA-boz.
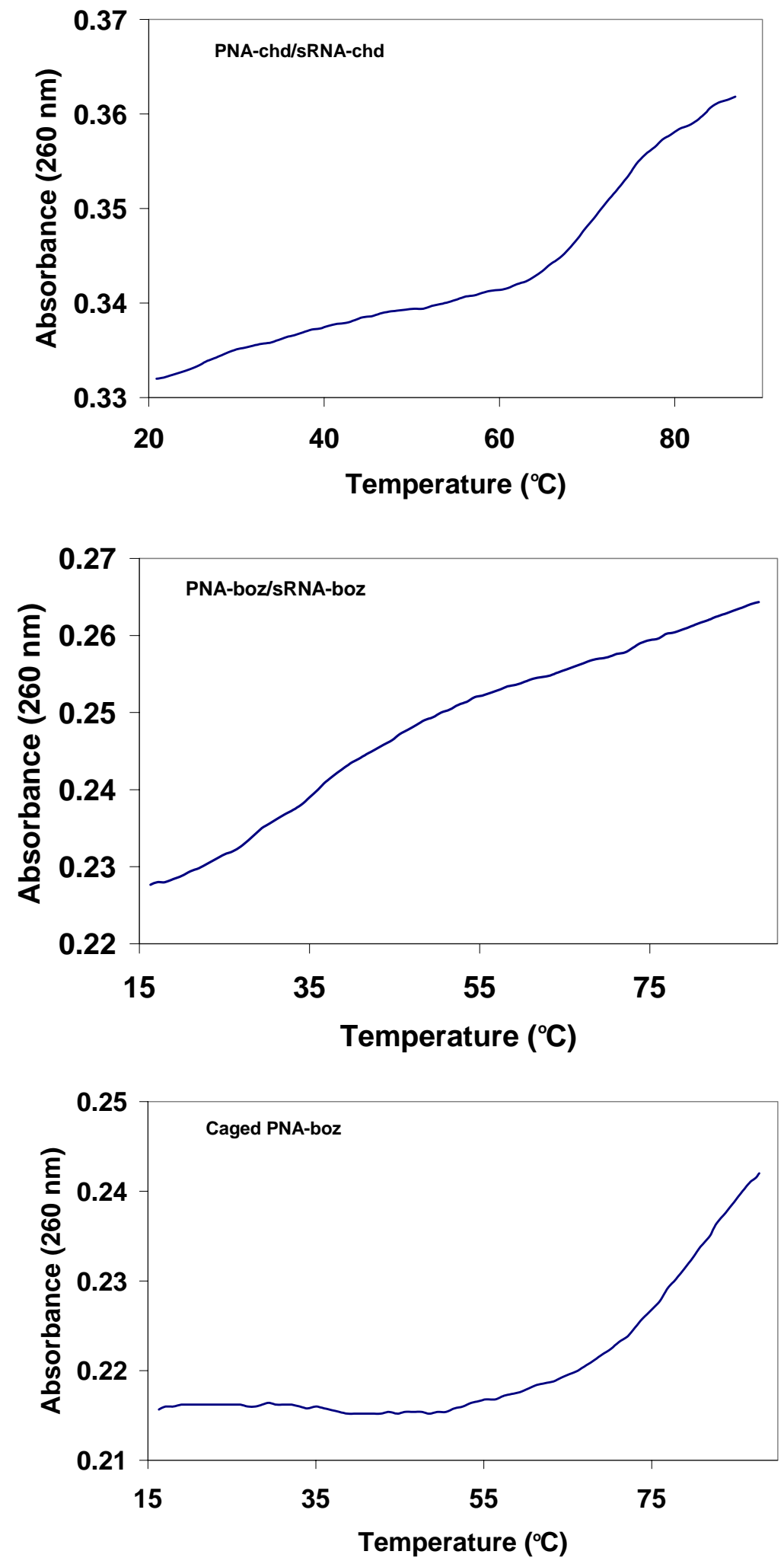
Figure S4. Structures of MB and caged PNA-chd and relative fluorescence intensity of MB, MB + PNA-chd, $\mathrm{MB}+$ caged PNA-chd, and MB + caged PNA-chd + 8 min photolysis (UV transilluminator, $9 \mathrm{~mW} / \mathrm{cm}^{2}$ at $365 \mathrm{~nm}$ ) at a concentration of $40 \mathrm{nM}$ in $10 \mathrm{mM}$ phosphate buffer at $30^{\circ} \mathrm{C}, \lambda_{\text {ex }}=488 \mathrm{~nm}$.

A
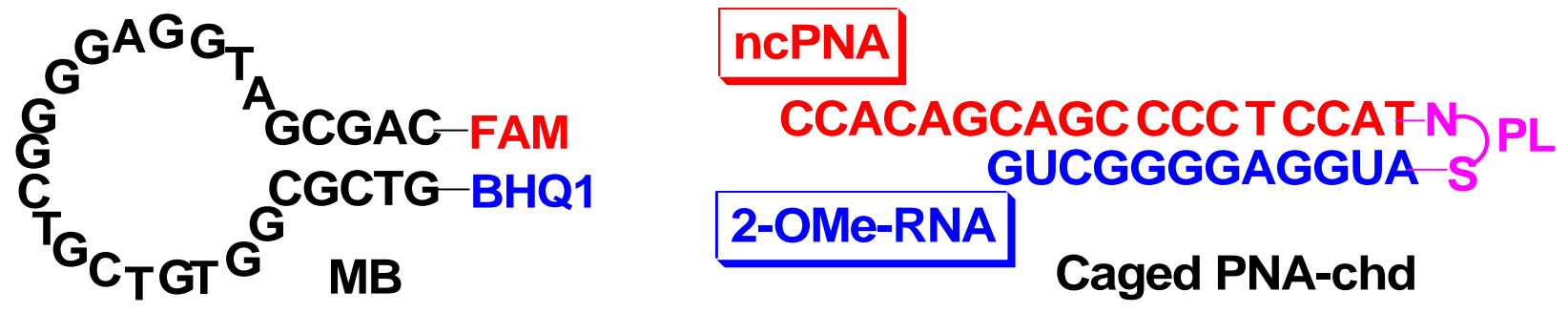

B

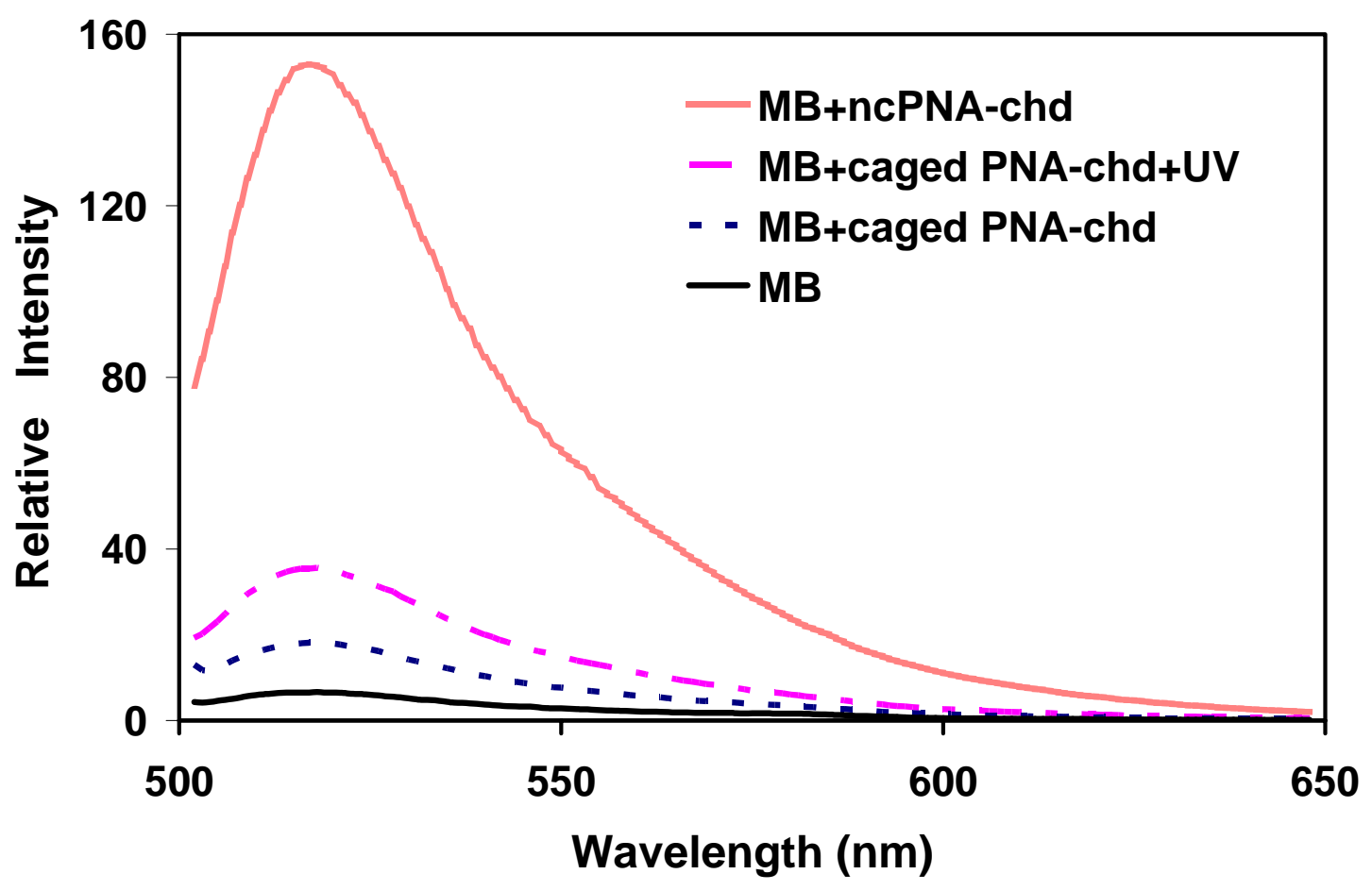


Figure S5. Photocleavage of caged ncPNA-PL-sDNA-Cy3 in vitro and in zebrafish embryos. Lane 1, sDNA-Cy3; Lane 2, ncPNA-PL-sDNA-Cy3; Lane 3, 8 min UV irradiation of ncPNAPL-sDNA-Cy3 in vitro; Lane 4, 8 min UV irradiation of ncPNA-PL-sDNA-Cy3 in zebrafish embryos.

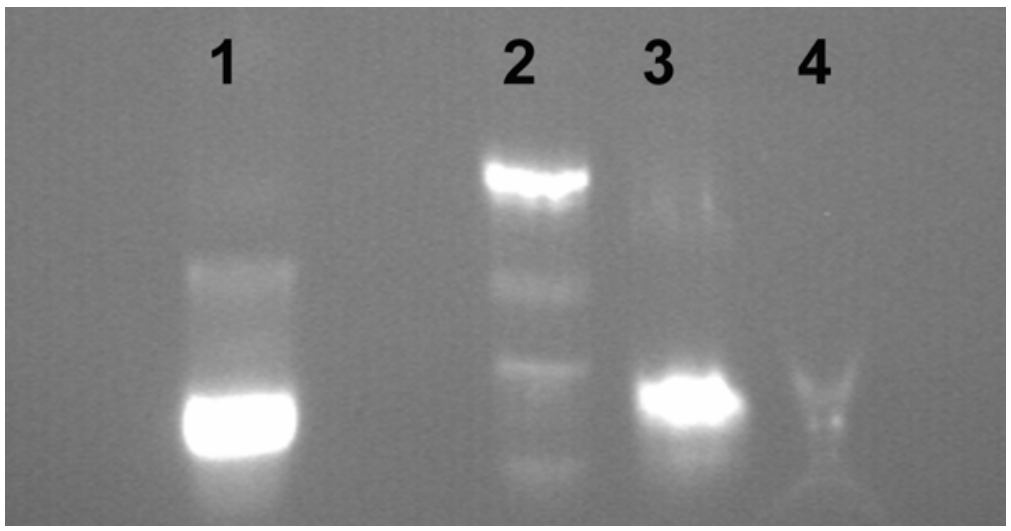

Figure S6. Zebrafish with varying degrees of "no-chordin" phenotype with different concentrations $(0,0.05,0.1,0.5 \mathrm{mM})$ of PNA-chd stock solutions injected (roughly $10 \mathrm{~nL}$ injected in each case). Embryos were incubated for $24 \mathrm{~h}$ at $28^{\circ} \mathrm{C}$, and then imaged by confocal microscopy. The uninjected ( $0 \mathrm{mM}$ PNA-chd) embryo also serves as a useful "normal" comparison for the embryo morphologies that were observed with caged ncPNAs, before and after UV activation.
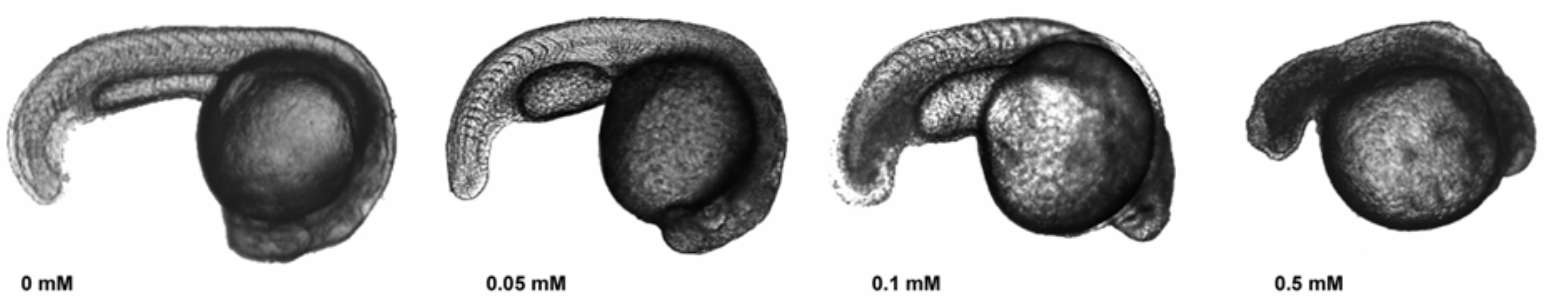
Figure S7. Transmitted light images of representative 24-hpf zebrafish embryos in experiments targeting bozozok mRNA. Photolysis conditions: UV transilluminator $9 \mathrm{~mW} / \mathrm{cm}^{2}$ at peak intensity, $365 \mathrm{~nm}$. A) Uninjected embryos UV-irradiated at $3 \mathrm{hpf}$ for 8 min developed normally. B) Injection with $0.5 \mathrm{mM}$ caged PNA-boz had no effect on embryos left in the dark. C) Embryos microinjected with $0.5 \mathrm{mM}$ PNA-boz exhibited the "no-bozozok" phenotype. D) Injection with $0.5 \mathrm{mM}$ caged PNA-boz and 8-min UV-irradiation at 2 hpf also gave the "no-bozozok" phenotype.
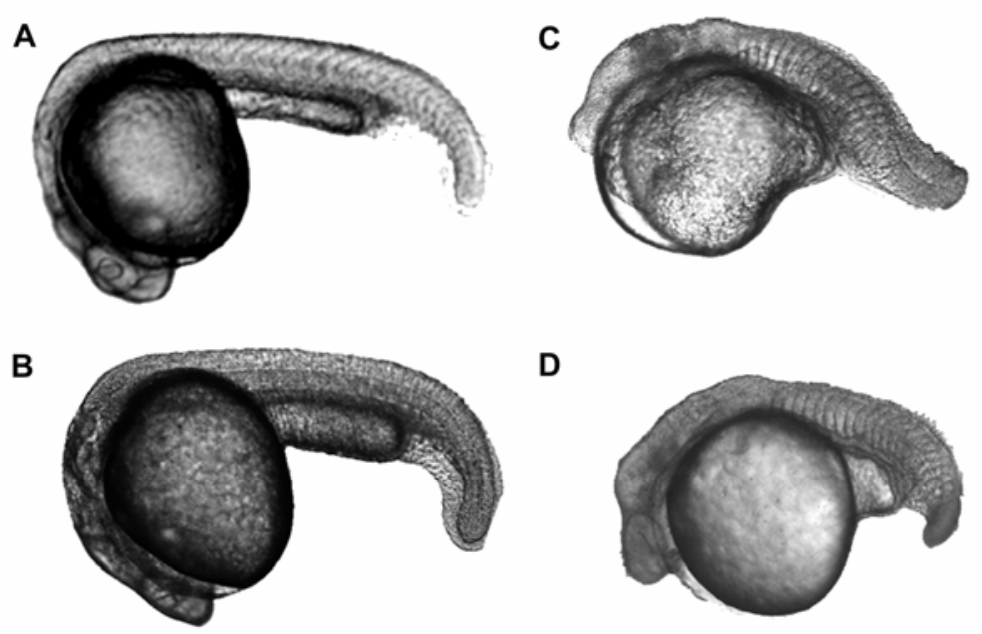

\section{Literature Cited}

(1) Maegawa, S.; Varga, M.; Weinberg, E. S. Development 2006, 133, 3265-3276.

(2) Tang, X.; Dmochowski, I. J. Nat. Protocols 2007, 1, 3041-3048; Tang, X.; Dmochowski, I. J. Angew. Chem., Int. Ed. 2006, 45, 3523-3526.

(3) From Integrated DNA Technologies (IDT), 5'-FAM-CAG CGA TGG AGG GGC TGC TGT GGC GCT G-BHQ1-3', where FAM is carboxyfluorescein, BHQ is black hole quencher, http://www.idtdna.com/Catalog/MolecularBeacon/Page1.aspx. 